\title{
Evidential network-based extension of Leaky Noisy-OR structure for supporting risks analyses
}

\author{
Fallet-Fidry G. ${ }^{1,2,3}$ - Weber P. ${ }^{2,3}$ - Simon C. ${ }^{2,3}$ - Iung B. ${ }^{2,3}$ - Duval C. ${ }^{1}$ \\ ${ }^{1}$ Electricité de France R\&D - Industrial Risk Management Department, Clamart, France \\ (e-mail: \{geoffrey.fallet;carole.duval\}@edf.fr) \\ ${ }^{2}$ Université de Lorraine, Centre de Recherche en Automatique de Nancy, UMR 7039, F-54506, France \\ (e-mail : \{geoffrey.fallet;philippe.weber;christophe.simon;benoit.iung\}@cran.uhp-nancy.fr) \\ ${ }^{3}$ CNRS, Centre de Recherche en Automatique de Nancy, UMR 7039, F-54506, France \\ (e-mail : \{geoffrey.fallet;philippe.weber;christophe.simon;benoit.iung\}@cran.uhp-nancy.fr)
}

\begin{abstract}
Bayesian Networks (BN) are used in risks analysis because their capacities allow supporting complex system modeling. Nevertheless, to achieve some modeling, one BN issue is still the effort required for quantification even if some solutions are addressing the use of logical structures like OR, AND, Noisy-OR, Leaky Noisy-OR, etc. These structures are useful to represent different uncertainties but they do not allow taking into account uncertainty on their parameters, logically present in risks analysis. To face this challenge, this paper aims at proposing imprecise extensions of the Leaky Noisy-OR structures and a solution to implement these imprecise structures by using Evidential networks.
\end{abstract}

Keywords: Bayesian Networks, Uncertainty, Noisy-OR, Leaky Noisy-OR, Evidential Networks

\section{INTRODUCTION}

\subsection{Bayesian Networks in Risks Analyses}

The Bayesian network (BN), initially developed to represent uncertain knowledge in artificial intelligence, is a probabilistic model allowing to take into account many variables with their dependencies and based on a graphical representation as described by Jensen (1996). The relationships between these variables are defined within Conditional Probability Tables (CPT). BN are also able to represent multiple-attributes correlated variables and to perform relevant simulations or diagnoses. As justified by Medina-Oliva et al. (2009), with these capacities leading to be well adapted to the modeling of complex systems, BN are more and more used in the field of reliability, safety or risks analysis of industrial systems. The first application example is shown in Hudson et al. (2001) and other ones are summarized in Medina-Oliva et al. (2009). In that way, it exists some equivalence with other well-known tools.

Industrial systems are complex due to the increasing number of components and their interactions. Moreover, risks analyses have to take into account different views such as technical, human, organizational and environmental ones. These areas include different types of correlated variables and data which can be both quantitative and qualitative. It is therefore necessary to be able to suitably represent these complex systems to make risks analyses more realistic and effective. In relation to an integrated vision, Léger et al. (2009) proposed the development of the Integrated Risks Analysis (IRA) based on the BN formalism and allowing taking into account technical, human, organizational and environmental items in the same approach to achieve risks analysis with simulations and diagnosis. Its objectives are: (1) prioritizing different risks, (2) helping the choice of barriers to reduce them, and (3) contributing to their control. In this type of risks analyses, it is required to deal with systems composed of many variables, many dependencies between them, and different types of data that can be uncertain (e.g. feedback data or experts' judgments). So, it is necessary to ensure the best possible representation of these variables to correctly handle the uncertainties leading to consistent results well adapted for their use by an analyst.

\subsection{Two main issues on data modeling within $B N$}

As mentioned by Antonucci (2011), one difficulty still open today in $\mathrm{BN}$ is the quantification step and more particularly the quantification of the Conditional Probability Tables (CPT). They define the conditional probability distribution on a child variable depending on all possible combination of the states of parent variables. However, the size of the CPT increases exponentially with the number of parent variables. For example, if a binary child variable $Y$ is depending on $n$ binary parents variables $X_{i}$ with two possible states ( $\mathrm{T}$ for "true" and $\mathrm{F}$ for "false") as in many risks analysis, the number of necessary values to completely define $\mathrm{P}\left(Y=\{\mathrm{T}\} \mid X_{i}\right)$ is equal to $2^{n}$. One solution is to assume a functional relationship to define the relation between parents variables and child variable as mentioned by Zagorecki and Druzdzel (2004). One of the most applied solution is the Noisy-OR (N$\mathrm{OR}$ ) and its extension called Leaky Noisy-OR (LN-OR). With these structures, fewer parameters are needed to define a CPT as shown by Pearl (1988) and Henrion (1989).

A second issue not fully solved on $\mathrm{BN}$ formalism is related to the uncertainties on variables and model parameters. Indeed, as argued by Zio (2009), it is necessary to be able to model, propagate and interpret uncertainty in risks analyses. Indeed, if the assessment of some variables can generally be done by using statistical distributions (e.g. based on feedback data), the assessment of some other variables is generally achieved 
with the use of experts' judgments. These different types of assessment involve different types of uncertainty. Two types of uncertainty are proposed by Hoffman and Hammonds (1994): the "random" uncertainty and the "epistemic" uncertainty. Random uncertainty corresponds to the natural variability of a physical phenomenon and epistemic uncertainty corresponds to a lack of knowledge. The use of experts' judgments raises the problem of the nature of uncertainty in risks studies. In several cases, it involves the use of other uncertainty modeling frameworks than the probabilistic framework as argued in Fallet et al. (2011). Even if structures like N-OR allow taking into account several types of uncertainty like uncertainty on dependencies or in the system modeling, it implies other sources of uncertainty. For example, the uncertainty on the parameters of these structures is not taking into account.

In that way, the objective of the work presented here is not to propose new modeling structures but to extend those existing and to enrich them by integrating other sources of uncertainty. More precisely, the paper aims at proposing imprecise N-OR and LN-OR structures for supporting several kinds of uncertainty. In that way, section II introduces N-OR and LN-OR structures and proposes a solution to implement them. Then, section III focuses on developments required to integrate several uncertainties in N-OR and LN-OR. The feasibility and added-value of these developments are shown in section IV for the case of the IRA methodology. Finally, conclusions and research perspectives are proposed on the basis of these different developments (section V).

\section{PROBLEM STATEMENT}

\subsection{Noisy-OR structure}

The Noisy-OR structure is an extension of the OR structure introduced by Pearl (1988) to reduce the elicitation effort in building $\mathrm{BN}$ for probabilistic models but also models that combine probability and logic. Noisy indicates that the causal interaction is not deterministic (any cause can produce the effect with some probability). Let us consider a binary variable $Y$ with $n$ binary parent variables $X_{i}$ (Fig. 1). These variables can be either true $\{\mathrm{T}\}$ or false $\{\mathrm{F}\} . X_{T}$ is defined as the set of $X_{i}$ which are "true" and $X_{F}$ is defined as the set of $X_{i}$ which are "false". The idea of Pearl is to associate to each $X_{i}$ a "link probability" $p_{i}$ such as $0 \leq p_{i} \leq 1$. The probability $p_{i}$ corresponds to the probability that $Y$ was true if $X_{i}$ is true. It illustrates the fact that the causal dependency between $X_{i}$ and $Y$ can be inhibited. This probability $p_{i}$ is defined as follows:

$$
p_{i}=\mathrm{P}\left(Y=\{\mathrm{T}\} \mid X_{i}=\{\mathrm{T}\}, X_{j, j \neq i}=\{\mathrm{F}\}\right)
$$

With this proposal, only $n$ parameters are sufficient to define completely the CPT of $Y$. Then, the probabilities that $Y$ will be "true" or "false" given $X_{i}$ are defined as follows:

$$
\begin{aligned}
& \mathrm{P}\left(Y=\{\mathrm{T}\} \mid X_{i}\right)=1-\prod_{X_{i} \in X_{T}}\left(1-p_{i}\right) \\
& \mathrm{P}\left(Y=\{\mathrm{F}\} \mid X_{i}\right)=\prod_{X_{i} \in X_{T}}\left(1-p_{i}\right)
\end{aligned}
$$

\subsection{Leaky Noisy-OR structure}

The N-OR structure implies that $Y$ is false with a probability equal to 1 if all its parent variables $X_{i}$ are false. However, in many cases, it is not a realistic assumption. Indeed, in several situations it is difficult to capture all the causes of $Y$ (e.g. for reliability purpose, it means to define all the failure modes of a component). Thus Henrion (1989) proposed an extension of the N-OR structure called Leaky Noisy-OR by introducing a new parameter called "leak probability". The leak probability corresponds to the fact that it may exist other parent variables to define more precisely the child variable. This leak probability can be modeled by using another parent variable $L$ with a link probability $p_{i}=l$ (Fig. 1). Let $l$ be this leak probability such as $0 \leq l<1$ and defined as follows:

$l=\mathrm{P}\left(Y=\{\mathrm{T}\} \mid X_{i}=\{\mathrm{F}\}, \forall i=1 \ldots n\right)$

Henrion (1989) proposed a first parameterization of the LNOR structure with $n+1$ parameters ( $n$ inhibition probability $p_{i}$ and one leak $l$ ):

$$
\begin{aligned}
& \mathrm{P}\left(Y=\{\mathrm{T}\} \mid X_{i}\right)=1-(1-l) \times \prod_{X_{i} \in X_{T}}\left(\frac{1-p_{i}}{1-l}\right) \\
& \mathrm{P}\left(Y=\{\mathrm{F}\} \mid X_{i}\right)=(1-l) \times \prod_{X_{i} \in X_{T}}\left(\frac{1-p_{i}}{1-l}\right)
\end{aligned}
$$

Diez (1993) gave another parameterization of the LN-OR structure as follow:

$$
\begin{aligned}
& \mathrm{P}\left(Y=\{\mathrm{T}\} \mid X_{i}\right)=1-(1-l) \times \prod_{X_{i} \in X_{T}}\left(1-p_{i}\right) \\
& \mathrm{P}\left(Y=\{\mathrm{F}\} \mid X_{i}\right)=(1-l) \times \prod_{X_{i} \in X_{T}}\left(1-p_{i}\right)
\end{aligned}
$$

These two parameterizations are mathematically equivalent but the difference is related to the question to be asked to the experts for knowledge elicitation. Henrion's parameterization led to a question like: "What is the probability that $Y$ is true given that $X_{i}$ is true and all other modeled variables are false?". Diez's parameterization is supported by a question like: "What is the probability that $Y$ is true given that $X_{i}$ is true and all other modeled and non-modeled variables are false?". In Henrion's parameterization, experts have to consider a combined influence of $X_{i}$ and the leak on $Y$. In Diez's parameterization, experts have to consider the link between $X_{i}$ and $Y$ with the leak absent. This paper is using the parameterization proposed by Diez (1993) by investigating imprecise extensions of this parameterization. Developments done in section 3 can be extended to Henrion's parameterization with the same proof than those used for Diez's parameterization.
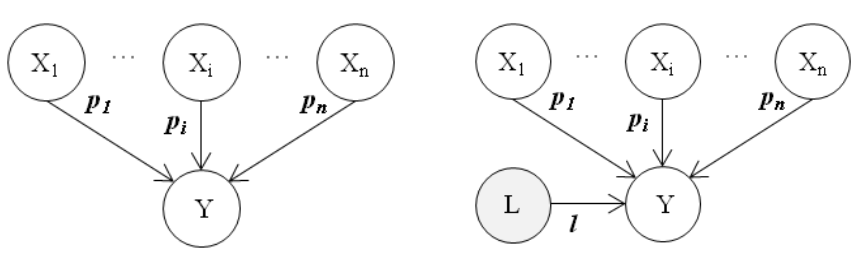

Fig. 1. Noisy-OR and Leaky Noisy-OR structures. 


\subsection{The problem of uncertainty in $\mathrm{N}-\mathrm{OR} / \mathrm{LN}-\mathrm{OR}$ structures}

The parameters defined in equations of sub-section 2.1 and 2.2 can be uncertain too. For example, the influence of a parent variable on a child variable but also the number of non-modeled parent variables or the state of parent variables can be not well-known. Indeed, in risks analyses, experts often use intervals to assess different variables. For example: an assessment by intervals can be used to assess the state of a parent variable or the influence of a parent variable on the child variable can be assessed with intervals. It is therefore necessary to be able to take into account uncertainties on the link probabilities, on the leak probability and on the state of parent variables $X_{i}$. Scientific contributions already exist about the problem of uncertainty in logical structures like $\mathrm{N}$ OR or LN-OR. Indeed, Srinivas (1993) and Diez (1993) proposed an extension of $\mathrm{N}-\mathrm{OR}$ structure for non-Boolean variables. Antonucci (2011) developed an imprecise LN-OR structure with uncertainty on the link probabilities that can be extended to the uncertainty on the leak probability (in Diez's parameterization). However, it exists only few works dealing with the problem of uncertainty on the state of Boolean parent variables. It can be noticed work of Simon and Weber (2009) for AND and OR gates. Moreover, there is no work dealing with the uncertainties on the parameters previously defined for $\mathrm{N}-\mathrm{OR}$ and $\mathrm{LN}-\mathrm{OR}$. Thus, the contribution finalized in this paper is to complete the previous works by proposing extensions of LN-OR structures to handle uncertainty on parameters and to reduce the effort in quantifying the CPT. In the next section, several extensions of generic relations of N-OR and LN-OR are investigated to take into account one or all of these uncertainties on parameters. In order to use them in risks analyses based on the $\mathrm{BN}$ formalism, a solution to implement imprecise N-OR and LN-OR is defined with the use of Evidential Networks.

\section{IMPRECISE N-OR AND LN-OR STRUCTURES}

\section{$3.1 \mathrm{LN}-\mathrm{OR}$ with uncertainty on the state of parent variables}

Simon and Weber (2009) have investigated a solution based on Evidential networks and Dempster-Shafer framework to take into account the uncertainty on the state of binary parent variables in AND and OR gates. Thus, the modeling formalization proposed by Simon and Weber is applied to the case of LN-OR gate. Let us consider variables $X_{i}$ that can be either $\{\mathrm{T}\}$ or $\{\mathrm{F}\}$. By considering an uncertainty on the state of $X_{i}$, another modality is defined: $\{\mathrm{T}, \mathrm{F}\}$. This modality defining the variable $X_{i}$ is exclusively in $\{\mathrm{T}\}$ or $\{\mathrm{F}\}$ state without distinguish exactly which. $X_{\mathrm{TF}}$ is defined as the set of $X_{i}$ in the state $\{\mathrm{T}, \mathrm{F}\}$. The value on the modality $\{\mathrm{T}, \mathrm{F}\}$ can be transferred to $\{\mathrm{T}\}$ or $\{\mathrm{F}\}$ if more information is available. Focusing on the case where $X_{\mathrm{i}}=\{\mathrm{T}, \mathrm{F}\}$, the first objective is to identify a bounding of $\mathrm{P}\left(Y=\{\mathrm{T}\} \mid X_{i}\right)$ and $\mathrm{P}\left(Y=\{\mathrm{F}\} \mid X_{i}\right)$ to characterize the uncertainty on $Y$ due to the uncertainty on the state of parent variables. Indeed, it is easy to proof that the uncertainty on the state of variable $X_{i}$ is equal to the difference between the lower and upper bounds as shown by Simon and Weber (2009). Lower bounds will correspond to the equations of $\mathrm{P}\left(Y=\{\mathrm{T}\} \mid X_{i}\right)$ and $\mathrm{P}\left(Y=\{\mathrm{F}\} \mid X_{i}\right)$. According to the generic relation of a $\mathrm{LN}-\mathrm{OR}$, it is necessary to maximize the product of $\left(1-p_{i}\right)$ for $X_{\mathrm{i}} \in X_{\mathrm{T}}$ in order to minimize $\mathrm{P}\left(Y=\{\mathrm{T}\} \mid X_{i}\right)$. On the contrary, it is necessary to minimize this product to minimize $\mathrm{P}\left(Y=\{\mathrm{F}\} \mid X_{i}\right)$. Let us consider that it exists $X_{\mathrm{k}}$ such as $X_{\mathrm{k}}=\{\mathrm{T}, \mathrm{F}\} . X_{\mathrm{k}}$ can be either $\{\mathrm{T}\}$ or $\{\mathrm{F}\}$. If $X_{\mathrm{k}}=\{\mathrm{T}\}$ then $X_{\mathrm{k}} \in X_{\mathrm{T}}$ and the product of $\left(1-p_{i}\right)$ for $X_{\mathrm{i}} \in X_{\mathrm{T}}$ decreases. Consequently, when at least one variable $X_{\mathrm{i}}$ such as $X_{\mathrm{i}} \in X_{\mathrm{TF}}$, the product of $\left(1-p_{i}\right)$ is minimized for $X_{\mathrm{i}} \in X_{\mathrm{T}}$ by taking into account variables $X_{\mathrm{i}}$ such as $X_{\mathrm{i}} \in X_{\mathrm{TF}}$ and the product is maximized by not taking into account variables $X_{\mathrm{i}}$ such as $X_{\mathrm{i}} \in X_{\mathrm{TF}}$, as shown in the following equations:

$$
\begin{aligned}
& \mathrm{P}\left(Y=\{\mathrm{T}\} \mid X_{i}\right)=1-(1-l) \times \prod_{X_{i} \in X_{T}}\left(1-p_{i}\right) \\
& \mathrm{P}\left(Y=\{\mathrm{F}\} \mid X_{i}\right)=(1-l) \times \prod_{X_{i} \in X_{T}}\left(1-p_{i}\right) \times \prod_{X_{i} \in X_{T F}}\left(1-p_{i}\right)
\end{aligned}
$$

The uncertainty on the state of $Y$ is defined as follow:

$$
\begin{aligned}
& \mathrm{P}\left(Y=\{\mathrm{T}, \mathrm{F}\} \mid X_{i}\right)=1-\mathrm{P}\left(Y=\{\mathrm{T}\} \mid X_{i}\right)-\mathrm{P}\left(Y=\{\mathrm{F}\} \mid X_{i}\right) \\
& =(1-l) \times \prod_{X_{i} \in X_{T}}\left(1-p_{i}\right) \times\left(1-\prod_{X_{i} \in X_{T F}}\left(1-p_{i}\right)\right)
\end{aligned}
$$

\subsection{LN-OR with uncertainty on the "link probabilities"}

Let us consider an uncertainty on link probabilities $p_{i}$. This parametric uncertainty can be modeled by an interval on $p_{i}$ with lower bound $p_{i \min }$ and upper bound $p_{i \max }$. By analyzing the derivate functions of the generic equations of the LN-OR structure, the bounds are defined for $\mathrm{P}\left(Y=\{\mathrm{T}\} \mid X_{i}\right)$ and $\mathrm{P}\left(Y=\{\mathrm{F}\} \mid X_{i}\right)$. The lower values of these bounds correspond to the relations of $\mathrm{P}\left(Y=\{\mathrm{T}\} \mid X_{i}\right)$ and $\mathrm{P}\left(Y=\{\mathrm{F}\} \mid X_{i}\right)$ when there is an uncertainty on $p_{i}$. With these bounds (defined in Appendix A.), the following relations are obtained:

$$
\begin{aligned}
& \mathrm{P}\left(Y=\{\mathrm{T}\} \mid X_{i}\right)=1-(1-l) \times \prod_{X_{i} \in X_{T}}\left(1-p_{i_{\text {min }}}\right) \\
& \mathrm{P}\left(Y=\{\mathrm{F}\} \mid X_{i}\right)=(1-l) \times \prod_{X_{i} \in X_{T}}\left(1-p_{i_{\text {max }}}\right)
\end{aligned}
$$

The uncertainty on the state of $Y$ is defined as follow:

$$
\begin{aligned}
& \mathrm{P}\left(Y=\{\mathrm{T}, \mathrm{F}\} \mid X_{i}\right)=1-\mathrm{P}\left(Y=\{\mathrm{T}\} \mid X_{i}\right)-\mathrm{P}\left(Y=\{\mathrm{F}\} \mid X_{i}\right) \\
& =(1-l) \times\left(\prod_{X_{i} \in X_{T}}\left(1-p_{i_{\text {min }}}\right)-\prod_{X_{i} \in X_{T}}\left(1-p_{i_{\max }}\right)\right)
\end{aligned}
$$

\subsection{LN-OR with uncertainty on the leak probability}

Let us consider an uncertainty on the leak probability $l$. This uncertainty can be modeled by an interval on $l$ with lower bound $l_{\min }$ and upper bound $l_{\max }$. By analyzing the derivate functions of the generic relations of the LN-OR structure, the bounds are defined for $\mathrm{P}\left(Y=\{\mathrm{T}\} \mid X_{i}\right)$ and $\mathrm{P}\left(Y=\{\mathrm{F}\} \mid X_{i}\right)$. The lower values of these bounds correspond to the relations of $\mathrm{P}\left(Y=\{\mathrm{T}\} \mid X_{i}\right)$ and $\mathrm{P}\left(Y=\{\mathrm{F}\} \mid X_{i}\right)$ when there is uncertainty on the link probability. Based on these bounds (defined in Appendix A.), the following relations are obtained:

$\mathrm{P}\left(Y=\{\mathrm{T}\} \mid X_{i}\right)=1-\left(1-l_{\min }\right) \times \prod_{X_{i} \in X_{T}}\left(1-p_{i}\right)$ 


$$
\mathrm{P}\left(Y=\{\mathrm{F}\} \mid X_{i}\right)=\left(1-l_{\max }\right) \times \prod_{X_{i} \in X_{T}}\left(1-p_{i}\right)
$$

The uncertainty on the state of $Y$ is defined as follow:

$$
\mathrm{P}\left(Y=\{\mathrm{T}, \mathrm{F}\} \mid X_{i}\right)=\left(l_{\text {max }}-l_{\text {min }}\right) \times \prod_{X_{i} \in X_{T}}\left(1-p_{i}\right)
$$

Let us consider the definition of the "leak probability". It corresponds to the fact that it may exist non-modeled parent variables. Consequently, if $l$ is equal to 0.05 , it makes more sense to consider $0 \leq l \leq 0.05$ because it is not sure that it exists non-modeled parent variables. In the LN-OR structure of Henrion, the leak is defined as the probability that $Y$ was true given that all $X_{\mathrm{i}}$ are false. With the imprecise LN-OR structure proposed in this section, the leak probability $l$ is defined as follows according to (15), (16) and (17):

$$
l=\mathrm{P}\left(Y=\{\mathrm{T}, \mathrm{F}\} \mid X_{i}=\{F\}, \forall i=1 \ldots n\right)
$$

This relation proposes another interpretation and modeling of $l$. The leak probability corresponds to an ignorance on the exhaustiveness of the parent variables. Indeed, this interpretation defined the leak as the fact that non-modeled parent variables may exist while the original definition of Henrion considers that non-modeled parent variables exist if $l \neq 0$. In section 4 , the difference between the interpretation of Henrion and the interpretation of (18) is shown.

\subsection{LN-OR with uncertainty on all parameters}

By using the relationships previously identified for each type of uncertainty on the LN-OR parameters, general relations taking into account the three kinds of uncertainty are defined. Then, the uncertainty in the LN-OR is integrated as follows:

$$
\begin{aligned}
& \mathrm{P}\left(Y=\{\mathrm{T}\} \mid X_{i}\right)=1-\left(1-l_{\text {min }}\right) \times \prod_{X_{i} \in X_{T}}\left(1-p_{i_{\text {min }}}\right) \\
& \mathrm{P}\left(Y=\{\mathrm{F}\} \mid X_{i}\right)=\left(1-l_{\text {max }}\right) \cdot \prod_{X_{i} \in X_{T}}\left(1-p_{i_{\text {max }}}\right) \cdot \prod_{X_{i} \in X_{T F}}\left(1-p_{i_{\max }}\right) \\
& \mathrm{P}\left(Y=\{\mathrm{T}, \mathrm{F}\} \mid X_{i}\right)=\left(1-l_{\text {min }}\right) \times \prod_{X_{i} \in X_{T}}\left(1-p_{i_{\min }}\right) \\
& -\left(1-l_{\text {max }}\right) \times \prod_{X_{i} \in X_{T}}\left(1-p_{i_{\max }}\right) \times \prod_{X_{i} \in X_{T F}}\left(1-p_{i_{\max }}\right)
\end{aligned}
$$

\subsection{Specialization to the Noisy-OR structure}

The relations identified in the previous sub-sections dedicated to handle uncertainty on the LN-OR parameters can be specialized to the characterization of the uncertainty on the parameters of the N-OR structure. For this purpose, a leak $l$ is considered equal to zero in (19), (20) and (21):

$$
\begin{aligned}
& \mathrm{P}\left(Y=\{\mathrm{T}\} \mid X_{i}\right)=1-\prod_{X_{i} \in X_{T}}\left(1-p_{i_{\min }}\right) \\
& \mathrm{P}\left(Y=\{\mathrm{F}\} \mid X_{i}\right)=\prod_{X_{i} \in X_{T}}\left(1-p_{i_{\max }}\right) \times \prod_{X_{i} \in X_{T F}}\left(1-p_{i_{\text {max }}}\right)
\end{aligned}
$$

The uncertainty on the state of $Y$ is defined as equal to:

$$
\begin{aligned}
& \mathrm{P}(Y=\{\mathrm{T}, \mathrm{F}\})= \\
& \prod_{X_{i} \in X_{T}}\left(1-p_{i_{\min }}\right)-\prod_{X_{i} \in X_{T}}\left(1-p_{i_{\max }}\right) \times \prod_{X_{i} \in X_{T F}}\left(1-p_{i_{\max }}\right)
\end{aligned}
$$

\subsection{From Bayesian Networks to Evidential Networks}

In order to use the relations defined in subsections 3.3 to 3.5 , it is important to find a relevant model to encode and to propagate these relations in a graphical representation. The use of the Evidential networks model proposed by Simon and Weber (2009) is taken as a solution to implement imprecise $\mathrm{LN}-\mathrm{OR}$ and to integrate them in Bayesian risks models. Evidential networks consist in a graphical representation of knowledge like BN but with belief masses instead of probabilities. As mentioned by Simon and Weber (2009), Evidential networks are based on the Dempster-Shafer framework and the Bayesian inference is extended to belief masses (corresponding to the probabilities defined in previous equations). The proposed Evidential networks formalism is applied to the performance and the reliability of systems. The uncertainty is represented by intervals with upper and lower bounds. As for a BN, an EN allow dealing with a lot of variables and modeling the dependences and the expression of relationships between the variables of the study on a graphical structure (Fig. 2). The difference between the two models is the underlying framework.

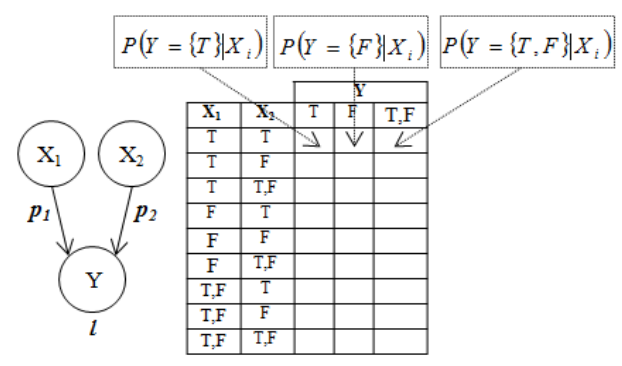

Fig. 2. Implementing imprecise LN-OR relations in CPTs

The next section is dedicated to the application of the imprecise LN-OR structure in risks analyses and more particularly in the Integrated Risks Analysis methodology by using Evidential networks. The objective is to show (a) how imprecise LN-OR structure can be used in risks analysis and (b) the interest of the developments proposed in section 3 .

\section{APPLICATION TO THE IRA METHODOLOGY}

\subsection{The use of Leaky Noisy-OR in the IRA methodology}

The LN-OR structure is used in the IRA methodology as explained by Léger et al. (2009). It allows to characterize the relation between the organizational, human and technical views in BN dedicated to the study of an industrial system. For instance, maintenance or operating actions are defined by a "barrier model" with organizational and human variables linked to the efficiency of the barrier as shown in Fig. 3. The links between the variables characterize the influence of a variable to another through the use of "aggravation factors" (noted $\alpha_{\mathrm{i}}$ ). For sake of illustration, this section consider a barrier model with only the human variables and the three phases of maintenance or operating actions (Preparation, Realization and Closing) and the action efficiency E. Each human indicator is either "true" or "false" and is assessed by an aggravation factor $\alpha_{i}$ that corresponds to its influence on a 
phase if it is "true". LN-OR structure allows building the CPT of each phase of maintenance or operating actions and the CPT for the efficiency of the studied barrier. The link probabilities $p_{i}$ corresponds to the aggravation factors $\alpha_{i}$. Aggravation factors characterize the worsening influence of the state of human indicators on the efficiency of the phase (or the worsening influence of the efficiency of a phase on the efficiency of the action). The leak probability corresponds to the fact that it may exist other variables to describe each phase of the action. Thus, the relations used to apply the LNOR to the IRA methodology are: $l=l$ and $\alpha_{i}=l-p_{i}$.

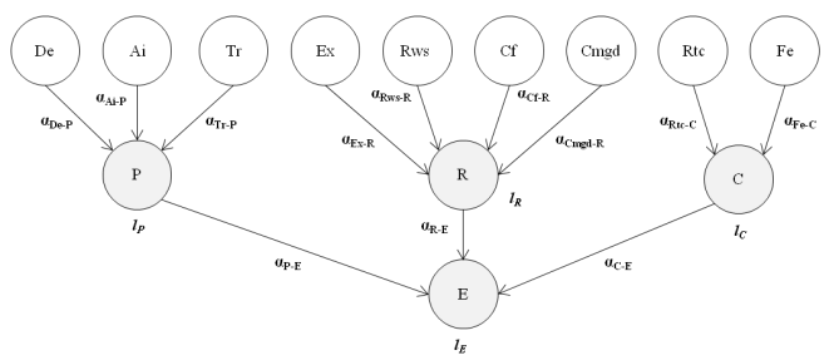

Fig. 3. IRA barrier model with LN-OR parameters

In the IRA methodology, there exist uncertainties on $l, \alpha_{\mathrm{i}}$ and the state of parent variables. Accordingly with (19) to (21), the relations for imprecise LN-OR is applied to the IRA methodology by replacing $p_{i}$ with $\alpha_{i}$ such as $\alpha_{i \text { min }}=1-p_{i \text { max }}$ and $\alpha_{i \max }=1-p_{i \min }$. Then, the CPT is defined by :

$$
\begin{aligned}
& \mathrm{P}\left(Y=\{\mathrm{T}\} \mid X_{i}\right)=1-\left(1-l_{\text {min }}\right) \times \prod_{X_{i} \in X_{T}} \alpha_{i_{m z c}} \\
& \mathrm{P}\left(Y=\{\mathrm{F}\} \mid X_{i}\right)=\left(1-l_{\text {max }}\right) \times \prod_{X_{i} \in X_{T}} \alpha_{i_{\min }} \times \prod_{X_{i} \in X_{T F}} \alpha_{i_{\min }} \\
& \mathrm{P}\left(Y=\{\mathrm{T}, \mathrm{F}\} \mid X_{i}\right)=\left(1-l_{\min }\right) \times \prod_{X_{i} \in X_{T}} \alpha_{i_{\max }} \\
& -\left(1-l_{\text {max }}\right) \times \prod_{X_{i} \in X_{T}} \alpha_{i_{\min }} \times \prod_{X_{i} \in X_{T F}} \alpha_{i_{\min }}
\end{aligned}
$$

\subsection{Application}

First, the preparation phase $P$ (Fig. 3) is analyzed to illustrate the use of the imprecise LN-OR in the IRA methodology. Let us consider that the preparation phase $P$ has a leak probability $l$ equals to 0.05 corresponding to the fact that it may exist other human indicators to define $P$. The study can be encoded in a risk model by using Evidential networks as mentioned in section 3.6. Three modalities are defined for each node: $\{\mathrm{T}\}$, $\{\mathrm{F}\},\{\mathrm{T}, \mathrm{F}\}$. To quantify each line of a CPT, (25), (26) and (27) are used as shown in Fig. 2.

For the first simulation, only an uncertainty on the leak probability $l$ is considered to underline the difference between the interpretation given by Henrion and those proposed in section 3.3. Let us consider $\alpha_{\mathrm{i}}=0.5$ for each link and prior distributions equal to $0.5 / 0.5$ for each human variable. By considering the leak equal to 0.05 , $\mathrm{P}(P=\{\mathrm{T}\})=0.5992$ is obtained. Considering the leak such as 0 $\leq l \leq 0.05$, the result is $\mathrm{P}(P=\{\mathrm{T}\}) \in[0.5781 ; 0.5992]$. This interval corresponds to the uncertainty due to the possible non-modeled causes. The upper bound characterizes the most positive situation when considering the leak definition.
Now, consider that the state of each human indicator is completely unknown (total ignorance). In the generic LN-OR (probabilistic framework), this ignorance is modeled by considering $\mathrm{P}\left(X_{i}=\{\mathrm{T}\}\right)=\mathrm{P}\left(X_{i}=\{\mathrm{F}\}\right)=0.5$ with the "indifference principle" proposed by Levine and Tribus (1978). With the imprecise LN-OR, this ignorance is modeled by considering the modality $\{\mathrm{T}, \mathrm{F}\}$ equals to 1 thanks to the "least commitment principle". Whereas $\mathrm{P}(P=\{\mathrm{T}\})=0.5992$ is obtained with the generic LN-OR, the result is $\mathrm{P}(P=\{\mathrm{T}\}) \in[0 ; 0.8812]$ with the imprecise LN-OR. This interval corresponds to the bias induced by the needed adaptation of the generic LN-OR to represent total ignorance. Indeed, by considering an equiprobability on $X_{i}$ to model total ignorance, there is no difference of representation with an objective equiprobability in the generic LN-OR.

These studies can be extended easily to a whole barrier model by considering the realization phase $R$, the closing phase $C$ and the efficiency $E$ of the studied action. Four imprecise LN-OR are used for each barrier (Fig. 3). As for one phase, lower and upper bounds are obtained for each node and characterize the uncertainty on each variable according to the uncertainties on prior distributions, link probabilities and leak probabilities. Finally, imprecise LN-OR is also applied on a whole IRA model with 4 barriers (16 LN-OR) and more than hundred variables. All barriers were defined as previously mentioned. Other nodes of the risks model were defined to take into account the uncertainty on barriers and propagate it through the model. At the end, intervals are obtained for several variables like the safety level or the system availability, etc. The bounds provide information about the scatter on the unique value obtain with the use of the generic LN-OR structure. Thanks to these intervals, the analyst can take more realistic decision according to the business policy and the risks he has to manage. Indeed, depending on the decision strategy, the analyst has to choose between the most conservative results, the less risky decision, etc.

\section{CONCLUSIONS}

This paper proposes imprecise LN-OR structure for supporting several kinds of uncertainty within them. These structures are enriched by considering other sources of uncertainty than the random uncertainty. The objective of these developments is to provide more general quantification structures that the initial ones. The new structures allow integrating uncertainty on the structures' parameters like dependencies influences, states of parent variables, etc. Different relations are proposed each type of uncertainty and take into account all uncertainties at the same time. A specialization to the N-OR is also given. During the development of the imprecise structures, another interpretation of the leak probability defined by Henrion (1989) has been investigated. This interpretation takes into account the fact that non modeled causes (corresponding to $l$ ) can exist or not. The comparison between these two interpretations underlines that Henrion's interpretation is less conservative than the one proposed. Whereas Henrion considers that non-modeled causes exist if $l \neq 0$, our contribution is considering that non-modeled causes can exist if $l \neq 0$. 
Then, Evidential networks are used to solve the problem of inference related to the use of imprecise structures. "Advanced" LN-OR structures defined in this paper were implemented by adapting the Bayesian models semantics with new modalities. Indeed, by using Bayesian inference in Evidential networks, simulations and diagnosis can be easily achieved and providing more informative values to the analyst with lower/upper bounds.

Finally, in order to validate the proposed developments, an application of the imprecise structures to the IRA methodology is shown. The application underlines the information provided by the use of lower/upper bounds to characterize the uncertainty on several variables. In order to propose other solutions to build networks with uncertainty, many investigations should be addressed as future developments: extension to Henrion's parameterization, generalization to $n$-ary variables, generalization of other structures, etc.

\section{REFERENCES}

Antonucci, A. (2011). The Imprecise Noisy-OR Gate, Proceedings of the $14^{\text {th }}$ International Conference on Information Fusion, 709-715, Chicago.

Diez, F.J. (1993). Parameter adjustment in Bayes networks. The generalized Noisy OR-gate, Proceedings of the Ninth Annual Conference on Uncertainty in Artificial Intelligence (UAI-93), 99-10, Washington, D.C..

Fallet, G., Duval, C., Simon, C., Weber, P. and Iung, B. (2011). Expert judgment collecting and modeling: Application to the Integrated Risks Analysis (IRA) methodology, Proceedings of the $3^{\text {rd }}$ International Workshop on Dependable Control of Discrete Systems, Saarbrücken, Germany.

Henrion, M. (1989). Some practical issues in constructing belief networks, Uncertainty in Artificial Intelligence 3, Elsevier Sciences Publisher, North Holland.

Hoffman, F.O. and Hammonds, J.S. (1994). Propagation of uncertainty in risk assessments: the need to distinguish between uncertainty due to lack of knowledge and uncertainty due to the variability, Risk Analyses, 14 (5), 707-712.

Hudson, L., Ware, B., Laskey, K. and Mahoney, S. (2001). An application of bayesian networks to antiterrorism risk management for military planners, Technical Report, Digital Sandbox, Inc.

Jensen, F.V. (1996). An Introduction to Bayesian Networks, London, UCL Press.

Léger, A., Weber, P., Levrat, E., Duval, C., Farret, R. and Iung, B. (2009). Methodological developments for probabilistic risk analyses of socio-technical systems, Journal of Risk and Reliability, 223 (4), 313-332.

Levine, R.D. and Tribus, M. (1978). The maximum entropy formalism, MIT Press.

Medina-Oliva, G., Weber, P., Simon, C. and Iung, B. (2009). Bayesian networks applications on dependability, risk analysis and maintenance, $2^{\text {nd }}$ IFAC Workshop on Dependable Control of Discret System, Bari, Italy.

Pearl, J. (1988). Probabilistic Reasoning in Intelligent Systems, Morgan Kaufmann Plubishers, USA.
Simon, C. and Weber, P. (2009). Evidential networks for reliability analysis and performance evaluation of systems with imprecise knowledge, IEEE Transactions on Reliability, 58 (1), 69-97.

Srinivas, S. (1993). A generalization of the Noisy-OR model, Ninth Annual Conference on Uncertainty in Artificial Intelligence (UAI-93), 208-215, Washington D.C..

Zagorecki, A. and Druzdzel, M. (2004). An empirical study of probability elicitation under Noisy-OR assumption, $17^{\text {th }}$ International Florida Artificial Intelligence Research Symposium Conference, Miami Beach, USA.

Zio, E. (2009). Reliability engineering: Old problems and new challenges, Reliability Engineering and System Safety, 94, 125-141, 2009.

\section{Appendix A. UNCERTAINTY ON $p_{i}$ AND $l$}

The uncertainty on link probabilities $p_{i}$ can be represented by $p_{i \min } \leq p_{i} \leq p_{i \max }$. By analyzing the derivate functions of $\mathrm{P}\left(Y=\{\mathrm{T}\} \mid X_{i}\right)$ and $\mathrm{P}\left(Y=\{\mathrm{F}\} \mid X_{i}\right)$, bounds can be defined.

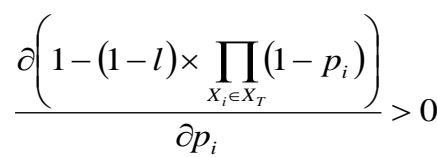

$$
\frac{\partial\left((1-l) \times \prod_{X_{i} \in X_{T}}\left(1-p_{i}\right)\right)}{\partial p_{i}}<0
$$

$\mathrm{P}\left(Y=\{\mathrm{T}\} \mid X_{i}\right)$ is increasing and its minimum $\underline{\mathrm{P}}$ is obtained for $p_{i}=p_{i \min }$ and its maximum $\overline{\mathrm{P}}$ for $p_{i}=p_{i \max }$. On the contrary, $\mathrm{P}\left(Y=\{\mathrm{F}\} \mid X_{i}\right)$ is decreasing and its minimum is obtained for $p_{i}=p_{i \max }$ and its maximum for $p_{i}=p_{i \min }$ :

$$
\begin{aligned}
& \underline{\mathrm{P}}\left(Y=\{\mathrm{T}\} \mid X_{i}\right)=1-(1-l) \times \prod_{X_{i} \in X_{T}}\left(1-p_{i_{\text {min }}}\right) \\
& \overline{\mathrm{P}}\left(Y=\{\mathrm{T}\} \mid X_{i}\right)=1-(1-l) \times \prod_{X_{i} \in X_{T}}\left(1-p_{i_{\text {max }}}\right) \\
& \underline{\mathrm{P}}\left(Y=\{\mathrm{F}\} \mid X_{i}\right)=(1-l) \times \prod_{X_{i} \in X_{T}}\left(1-p_{i_{\text {max }}}\right) \\
& \overline{\mathrm{P}}\left(Y=\{\mathrm{F}\} \mid X_{i}\right)=1-(1-l) \times \prod_{X_{i} \in X_{T}}\left(1-p_{i_{\text {min }}}\right) \\
& \mathrm{P}\left(Y=\{\mathrm{T}, \mathrm{F}\} \mid X_{i}\right)=1-\underline{\mathrm{P}}\left(Y=\{\mathrm{T}\} \mid X_{i}\right)-\underline{\mathrm{P}}\left(Y=\{\mathrm{F}\} \mid X_{i}\right)
\end{aligned}
$$

The uncertainty on the leak $l$ can be represented by $l_{\text {min }} \leq l \leq$ $l_{\text {max }}$. By analyzing the derivate functions of $\mathrm{P}\left(Y=\{\mathrm{T}\} \mid X_{i}\right)$ and $\mathrm{P}\left(Y=\{\mathrm{F}\} \mid X_{i}\right)$, bounds can be defined.

$$
\frac{\partial\left(1-(1-l) \times \prod_{X_{i} \in X_{T}}\left(1-p_{i}\right)\right)}{\partial l}>0 \frac{\partial\left((1-l) \times \prod_{X_{i} \in X_{T}}\left(1-p_{i}\right)\right)}{\partial l}<0
$$

$\mathrm{P}\left(Y=\{\mathrm{T}\} \mid X_{i}\right)$ is increasing and its minimum is obtained for $l=l_{\min }$ and its maximum for $l=l_{\max }$. On the contrary, $\mathrm{P}\left(Y=\{\mathrm{F}\} \mid X_{i}\right)$ is decreasing and its minimum is obtained for $l=l_{\max }$ and its maximum for $l=l_{\text {min }}$ :

$$
\begin{aligned}
& \underline{\mathrm{P}}\left(Y=\{\mathrm{T}\} \mid X_{i}\right)=1-\left(1-l_{\text {min }}\right) \times \prod_{X_{i} \in X_{T}}\left(1-p_{i}\right) \\
& \overline{\mathrm{P}}\left(Y=\{\mathrm{T}\} \mid X_{i}\right)=1-\left(1-l_{\text {max }}\right) \times \prod_{X_{i} \in X_{T}}\left(1-p_{i}\right) \\
& \underline{\mathrm{P}}\left(Y=\{\mathrm{F}\} \mid X_{i}\right)=\left(1-l_{\text {max }}\right) \times \prod_{X_{i} \in X_{T}}\left(1-p_{i}\right) \\
& \overline{\mathrm{P}}\left(Y=\{\mathrm{F}\} \mid X_{i}\right)=1-\left(1-l_{\text {min }}\right) \times \prod_{X_{i} \in X_{T}}\left(1-p_{i_{i}}\right)
\end{aligned}
$$

The uncertainty is defined in the same way as for $p_{i}$. 